DOI: $10.15593 / 2224-9982 / 2021.64 .11$

УДК $519.9,629.7$

М.Ю. Егоров ${ }^{1}$, Д.М. Егоров ${ }^{2}$, С.М. Егоров ${ }^{2}$

${ }^{1}$ Пермский национальный исследовательский политехнический университет, Пермь, Россия

${ }^{2}$ Научно-исследовательский институт полимерных материалов, Пермь, Россия

ЧИСЛЕННОЕ ИССЛЕДОВАНИЕ ДИНАМИКИ ВНУТРИКАМЕРНЫХ ПРОЦЕССОВ В МАРШЕВОМ РАКЕТНОМ ДВИГАТЕЛЕ НА ТВЕРДОМ

ТОПЛИВЕ С УЧЕТОМ ПОЛЕТНЫХ ПЕРЕГРУЗОК.

ЧАСТЬ 2. РЕЗУЛЬТАТЫ РАСЧЕТА

\begin{abstract}
Приводятся результаты численных расчетов динамики внутрикамерных процессов в маршевом РДТТ второй ступени крылатой ракеты с учетом распределенных пространственно-трехмерных и изменяющихся во времени полетных перегрузок, полученные с использованием разработанной ранее методики расчета и созданного на ее основе пакета прикладных программ. Величина полетной перегрузки крылатой ракеты в проекциях по осям координат определяется в зависимости от массы, скорости и траектории движения ракеты, массового расхода и тяги ракетного двигателя. Внешним аэродинамическим воздействием на внутрикамерный процесс в РДТТ пренебрегаем. Результаты расчетов приводятся в сравнении - без учета и с учетом действия полетной перегрузки. Рассматривается два основных этапа работы ракетного двигателя: выход на режим работы и маршевый режим работы. На этапе выхода на режим работы РДТТ перегрузка влияет на перераспределение температуры газовой фазы продуктов сгорания в районе переднего днища камеры сгорания. На маршевом режиме работы РДТТ в результате действия полетной перегрузки наблюдается увеличение плотности частиц (жидких капель) малого и большого диаметра твердой фазы продуктов сгорания на стенке заднего днища и предсоплового газохода, причем степень изменения распределения плотности частиц (жидких капель) большего диаметра более значительная. Результаты численного исследования хорошо согласуются с экспериментальными данными - результатами стендовых испытаний ракетного двигателя и результатами летных испытаний крылатой ракеты, в состав которой входит рассматриваемый РДТТ.

Ключевые слова: ракетный двигатель твердого топлива, горение твердого топлива, газовая динамика, учет полетных перегрузок, результаты численных расчетов.
\end{abstract}

\author{
M.Yu. Egorov' ${ }^{1}$ D.M. Egorov², S.M. Egorov ${ }^{2}$ \\ ${ }^{1}$ Perm National Research PolytechnicUniversity, Perm, Russian Federation \\ ${ }^{2}$ Research Institute of polymer materials, Perm, Russian Federation
}

\title{
NUMERICAL STUDY OF DYNAMICS INTRACHAMBER PROCESSES IN SOLID PROPELLANT SUSTAINER TAKING INTO ACCOUNT FLIGHT OVERLOADS.
}

\author{
PART 2. CALCULATION RESULTS
}

\begin{abstract}
The article presents the results of numerical calculations of the dynamics of in-chamber processes in the cruise missile's second-stage cruise missile propulsion system, taking into account the distributed space-three-dimensional and time-varying flight overloads, obtained using the previously developed calculation method and the application software package created on its basis. The value of the flight overload of a cruise missile, in projections along the coordinate axes, is determined depending on the mass, speed and trajectory of the rocket, mass flow and thrust of the rocket engine. The external aerodynamic effect on the in-chamber process in the SRM is neglected. The results of the calculations are given in comparison-without taking into account and taking into account the effect of flight overload. Two main stages of the rocket engine operation are considered: entering the operating mode and the main operating mode. At the stage of entering the SRM operation mode, the overload affects the redistribution of the temperature of the gas phase of the combustion products in the area of the front bottom of the combustion chamber. On the March operation of the SRB as a result of the flight of an overload, an increase of the particle density (liquid drops) small and large diameter of the solid phase of the combustion products in the wall of the back plate and pre-exhaust gas flue, and the degree of change in the distribution density of the particles (liquid droplets) that are larger than large. The results of the numerical study are in good agreement with the experimental data - the results of bench tests of the rocket engine and the results of flight tests of the cruise missile, which includes the considered SRM.

Keywords: solid propellant rocket engine, burning of solid propellant, gas dynamics, accounting for flight overloads, results of numerical calculations.
\end{abstract}




\section{Вводные замечания}

Приведем результаты численных расчетов динамики внутрикамерных процессов маршевого РДТТ второй ступени крылатой ракеты с учетом распределенных пространственно-трехмерных и изменяющихся во времени полетных перегрузок, полученные с использованием разработанной ранее методики расчета (уровня постановки вычислительного эксперимента) и созданного на ее основе пакета прикладных программ, приведенных в предыдущей статье (часть 1) (см. также дополнительно работы [1-5]).

Предварительно уточним некоторые расчетные позиции.

Вектор тяги рассматриваемого РДТТ изменяется по величине, но не изменяется по направлению (конструктивные ограничения). Маневрирование ракетой, в состав которой входит ракетный двигатель, осуществляется только за счет аэродинамических органов управления. Полет крылатой ракеты происходит на высоте более 12000 м. Плотность воздуха на данной высоте незначительная $\left(\rho \approx(0,3 \ldots 0,2) \mathrm{\kappa} / \mathrm{m}^{3}\right)$ [6]. Корпус ракеты имеет хорошо обтекаемые формы, т.е. имеет низкий коэффициент лобового сопротивления $\left(c_{x} \leq 0,1\right)$ [7]. С учетом вышеизложенного внешним аэродинамическим воздействием на внутрикамерный процесс в РДТТ пренебрегаем.

Суммарную величину полетных перегрузок разложим по осям координат, связанным с ракетным двигателем. Ось $0 Z$ совместим с продольной осью РДТТ. Начало координат поместим в районе переднего днища ракетного двигателя. Положительные направления осей $0 X, 0 Y$ и $0 Z$ ориентируем как правую тройку векторов, если смотреть на начало координат со стороны сопла.

Величина продольной полетной перегрузки (вдоль оси $0 Z$ ) определяется как отношение текущей тяги РДТТ к текущему весу ракеты [8]:

$$
N Z=\frac{R}{(m-\dot{m} t) g}
$$

Величина предельных боковых перегрузок (например, вдоль оси 0Y) при перемещении (маневрировании) крылатой ракеты по дуге окружности в нижней части своей траектории определяется по источнику [8]:

$$
N Y=\frac{m\left(g+\frac{v^{2}}{r}\right)}{(m-\dot{m} t) g} .
$$

В формулах (1), (2) приняты обозначения: $R$ - тяга РДТТ, $m$ - начальная масса ракеты, $\dot{m}$ - массовый расход РДТТ, $t-$ время, $g$ - ускорение свободного падения, $v$ - скорость ракеты, $r$ - радиус окружности.

Рассмотрим два основных этапа работы ракетного двигателя: выход на режим работы и маршевый режим работы. Все приведенные ниже функциональные параметры РДТТ будут даны в безразмерном виде.

\section{Выход на режим работы РДТТ}

Приведем некоторые результаты численных расчетов динамики внутрикамерных процессов на этапе выхода ракетного двигателя на режим работы. Рассмотрим данный режим работы РДТТ без учета и с учетом действия полетных перегрузок (распределенного по пространству и времени поля силы тяжести). Принципиальная компоновочная схема РДТТ на данном этапе его работы показана на рис. 1.

На рис. 2 приведен закон изменения величины полетных перегрузок в проекциях по осям координат $(N X=0, N Y=$ var, $N Z=$ var $)$ по времени работы РДТТ. Закон получен на основании выражений (1), (2) с учетом изменения тяги, массового расхода ракетного двигателя, а также с учетом скорости движения и радиуса разворота крылатой ракеты.

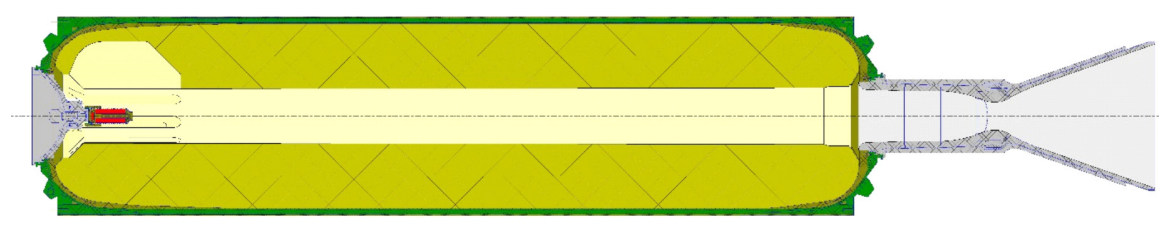

Рис. 1. Компоновочная схема РДТТ на этапе выхода на режим работы 


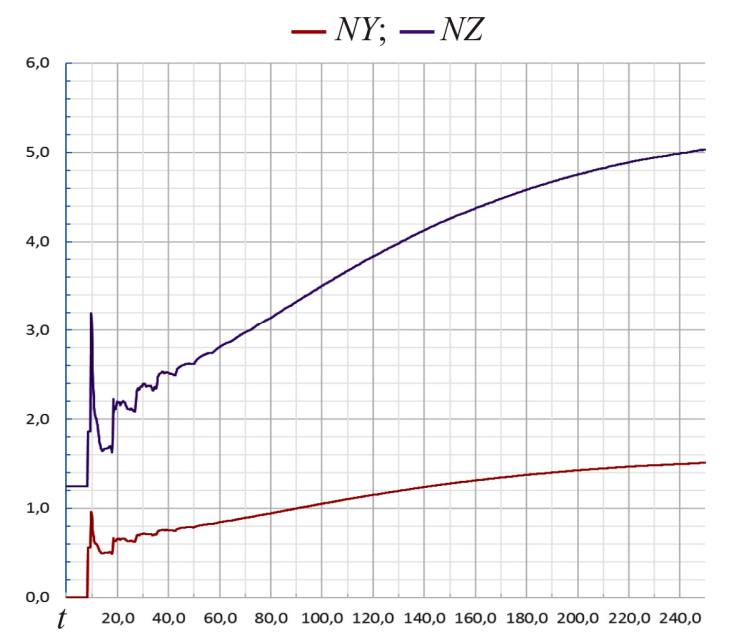

Рис. 2. Изменение величины полетных перегрузок

На рис. 3 представлено изменение во времени температуры газовой фазы продуктов сгорания - первой фазы гомогенно-гетерогенной смеси (см. предыдущую статью, часть 1) в фиксированных точках камеры сгорания (T1 переднее днище, Т2 - зона вершины щелей заряда твердого топлива, Т5 - заднее днище), соответственно без учета действия перегрузки (см. рис. $3, a$ ) и с учетом действия перегрузки (см. рис. 3,6 ). Из рисунков видно, что изменение во времени температуры газовой фазы продуктов сгорания в районе переднего днища РДТТ (Т1) различное. Наличие поля значительных продольных перегрузок (NZ) препятствует в этой зоне глубокому перемешиванию низкотемпературного воздуха и высокотемпе- ратурных продуктов сгорания пиротехнического воспламенительного состава и смесевого твердого топлива. Это, в свою очередь, отражается на характере прогрева данной области камеры сгорания ракетного двигателя, что приводит к некоторой задержке прогрева и зажигания поверхности горения заряда смесевого твердого топлива в канально-щелевой его части (ближе к переднему днищу). Следует отметить также различие в изменении температуры газовой фазы продуктов сгорания в районе заднего днища (Т5) в начальный момент времени работы РДТТ.

На рис. 4 показано распределение в камере сгорания и сопловом блоке ракетного двигателя температуры газовой фазы продуктов сгорания в фиксированный момент времени $(t=250)$ в плоскости $Y 0 Z$ соответственно, без учета действия перегрузки (см. рис. $4, a)$ и с учетом действия перегрузки (см. рис. 4, б). За счет действия перегрузки поле температуры газовой фазы продуктов сгорания в камере сгорания как бы выравнивается, разглаживается - становится более равномерным.

В меньшей степени эффект выравнивания касается распределения в камере сгорания и сопловом блоке РДТТ модуля вектора скорости газовой фазы продуктов сгорания в фиксированный момент времени $(t=250)$ в плоскости $Y 0 Z$ соответственно, без учета действия перегрузки (рис. $5, a$ ) и с учетом действия перегрузки (рис. 5, б).

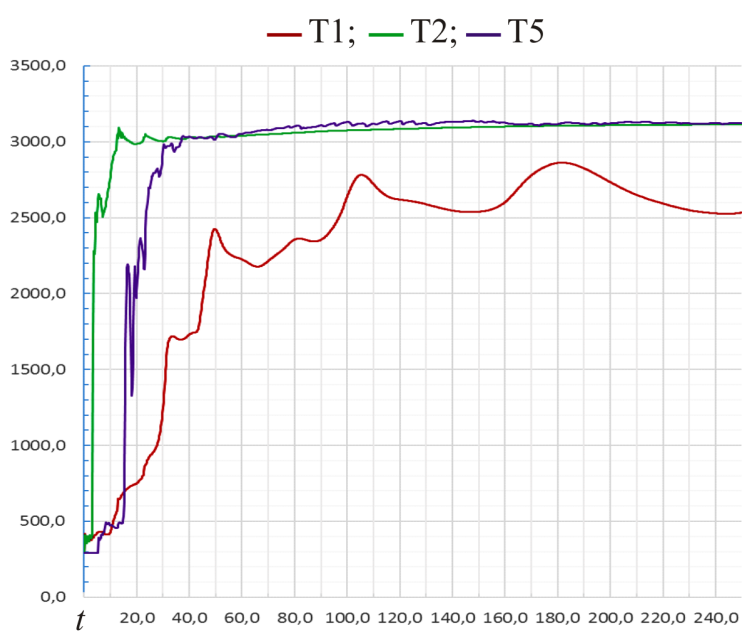

$a$

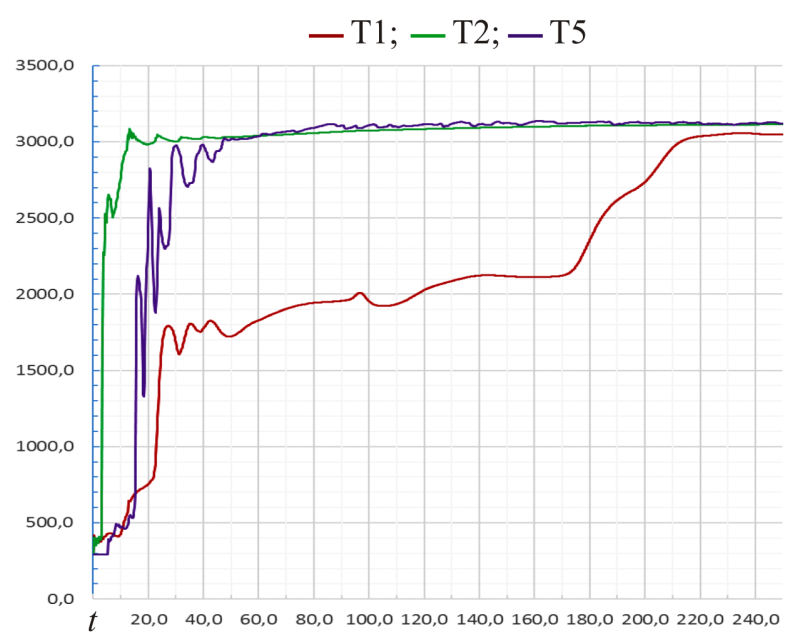

б

Рис. 3. Изменение во времени температуры газовой фазы продуктов сгорания: $a$ - без учета действия перегрузки; $\sigma$ - с учетом действия перегрузки 


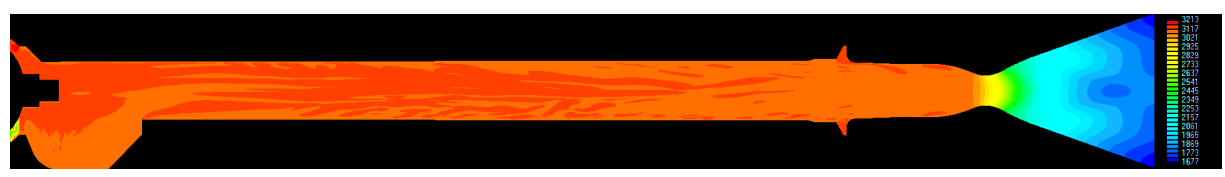

$a$

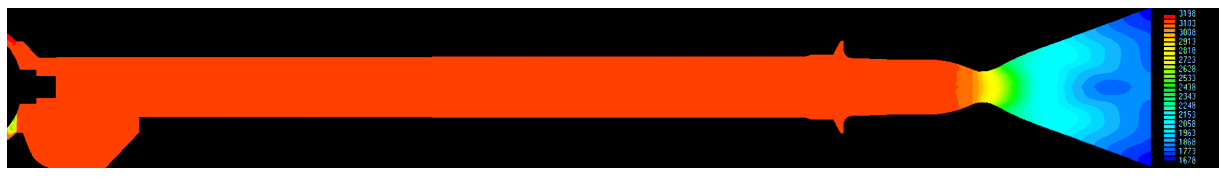

6

Рис. 4. Распределение температуры газовой фазы продуктов сгорания в фиксированный момент времени $(t=250)$ в плоскости $Y 0 Z: a-$ без учета действия перегрузки; $\sigma$ - с учетом действия перегрузки

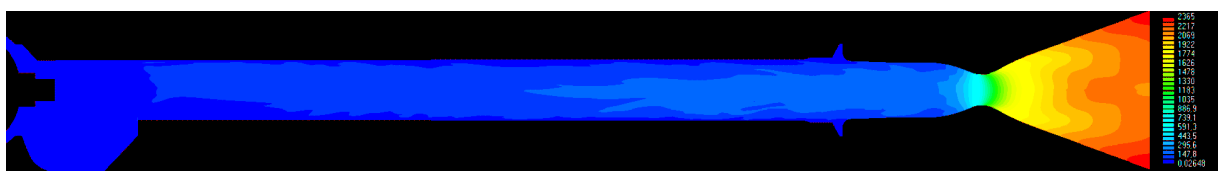

$a$

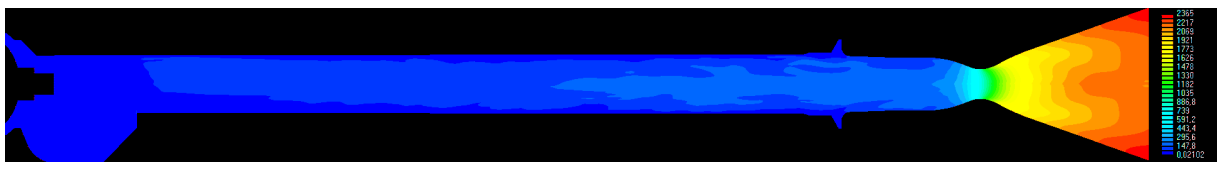

$\sigma$

Рис. 5. Распределение модуля вектора скорости газовой фазы продуктов сгорания в фиксированный момент времени $(t=250)$ в плоскости $Y 0 Z$ :

$a$ - без учета действия перегрузки; $\sigma$ - с учетом действия перегрузки

Изменение параметров других компонентов гомогенно-гетерогенной смеси продуктов сгорания (мелкодисперсные несгоревшие частицы в продуктах сгорания пиротехнического воспламенительного состава - вторая фаза смеси, мелкодисперсные несгоревшие частицы малого диаметра в продуктах сгорания заряда смесевого твердого топлива - третья фаза смеси и мелкодисперсные несгоревшие частицы большого диаметра в продуктах сгорания заряда смесевого твердого топлива - четвертая фаза смеси (см. предыдущую статью, часть 1)) на данном режиме работы ракетного двигателя без учета действия перегрузки и с учетом действия заданной перегрузки незначительные.

В целом влияние поля силы тяжести, даже на этапе выхода РДТТ на режим работы при относительно невысоком уровне перегрузок, заметно. По мере выгорания свода заряда смесевого твердого топлива уровень полетных перегрузок возрастает, так как уменьшается масса крылатой ракеты и существенно увеличивается ее скорость движения.

\section{Маршевый режим работы РДТТ}

Приведем некоторые результаты численных расчетов динамики внутрикамерных процессов на маршевом режиме работы РДТТ. Рассмотрим данный режим работы ракетного двигателя без учета и с учетом действия полетных перегрузок (распределенного по пространству и времени поля силы тяжести). Принципиальная компоновочная схема РДТТ на маршевом режиме его работы показана на рис. 6.

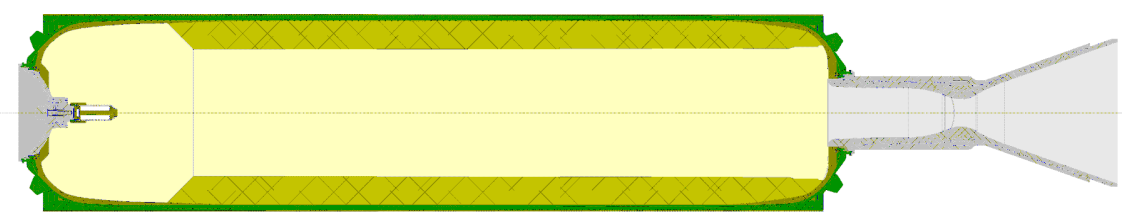

Рис. 6. Компоновочная схема РДТТ на маршевом режиме работы 
На рассматриваемом режиме работы ракетного двигателя изменением по времени величины полетных перегрузок в проекциях по осям координат можно пренебречь ( $N X=$ const, $N Y=$ const, $N Z=$ const), так как постоянная времени камеры сгорания РДТТ ( $\left.\bar{t}=\frac{m}{\dot{m}}\right)$ в данном случае существенно меньше времени, в течение которого это изменение происходит. Кроме того, на этом режиме работы тяга ракетного двигателя и скорость движения крылатой ракеты изменяются незначительно (см. выражения (1), (2)).

На рис. 7 дано распределение в камере сгорания и сопловом блоке РДТТ полной удельной энергии газовой фазы продуктов сгорания в фиксированный момент времени в плоскости $Y 0 Z$ соответственно без учета действия перегрузки (см. рис. 7, $a: N X=0$, $N Y=0, N Z=0)$ и с учетом действия перегрузки на двух режимах (см. рис. 7, б: $N X=0$, $N Y=0,3, N Z=10$ и рис. $7,6: N X=0, N Y=20$, $N Z=10)$.

На рис. 8 представлено распределение в камере сгорания и сопловом блоке ракетного двигателя энтропийной функции $\left(S=\frac{p}{\rho^{k}}\right)$ газовой фазы продуктов сгорания в фиксированный момент времени в плоскости $Y 0 Z$ на тех же режимах ввода перегрузки, что и на рис. 7. Здесь в приведенной формуле $p$ - давление, $\rho-$ плотность и $k-$ показатель адиабаты.
Анализируя данные рис. 7, 8, можно сделать вывод о том, что параметры газовой фазы продуктов сгорания под действием наложенной перегрузки изменяются (особенно по полной удельной энергии в районе переднего днища), но несущественно. Рисунки различны в локальных деталях. В целом характер распределения остается тот же. Можно говорить о малой инерционности газовой фазы продуктов сгорания из-за относительно низкой ее плотности, особенно в зонах торможения (район переднего и заднего днищ).

На рис. 9 показано распределение в камере сгорания и сопловом блоке РДТТ плотности мелкодисперсных несгоревших частиц (жидких капель) малого диаметра ( $d_{3}=2$ мкм) в продуктах сгорания заряда смесевого твердого топлива (третья фаза гомогенно-гетерогенной смеси) в фиксированный момент времени в плоскости $Y 0 Z$ на тех же режимах ввода перегрузки, что и на рис. 7,8 . Дополнительно на рис. 10 дано то же распределение, но в плоскости $X 0 Y$ в районе заднего днища. Из рисунков видно, что распределение мелкодисперсных несгоревших частиц малого диаметра, особенно в районе заднего днища и центрального предсоплового газохода, в зависимости от уровня и характера перегрузок меняется существенно. При действии перегрузки твердая (жидкая) фаза частиц как бы размазывается по стенке заднего днища и предсоплового газохода. Причем чем

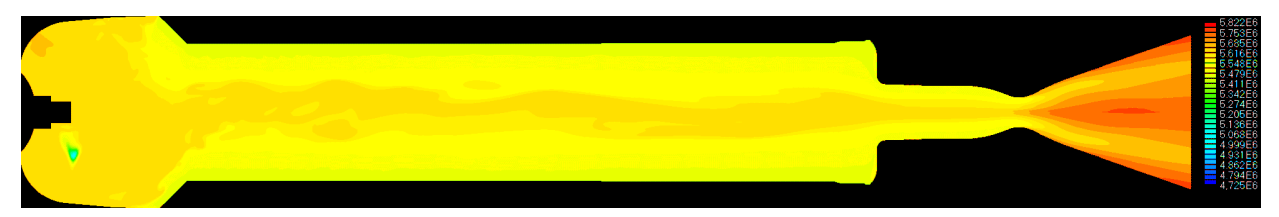

$a$

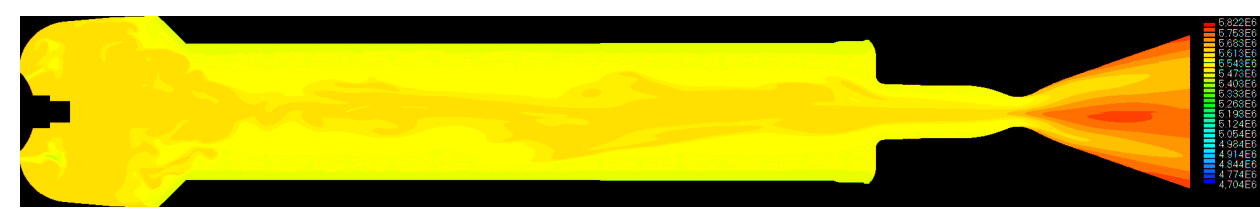

6

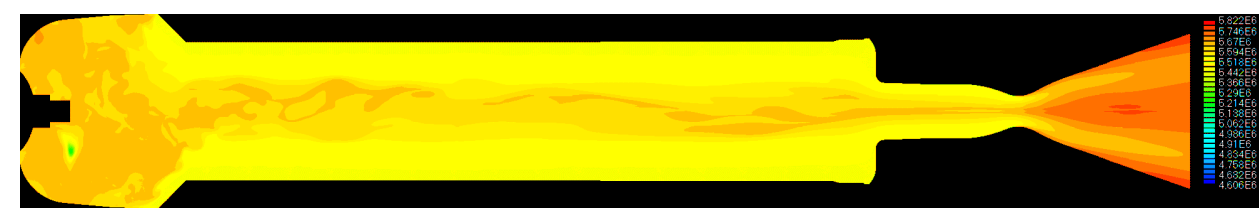

B

Рис. 7. Распределение полной удельной энергии газовой фазы в плоскости $Y 0 Z$ : $a-N X=0, N Y=0, N Z=0 ; \sigma-N X=0, N Y=0,3, N Z=10 ; в-N X=0, N Y=20, N Z=10$ 


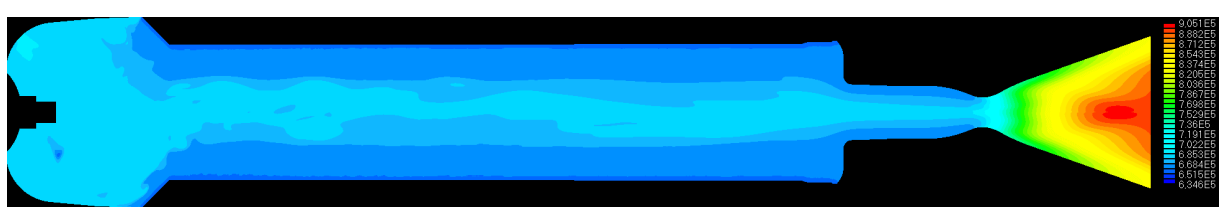

$a$

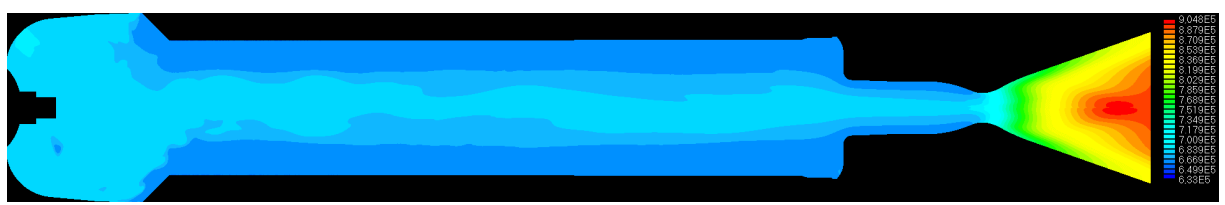

б

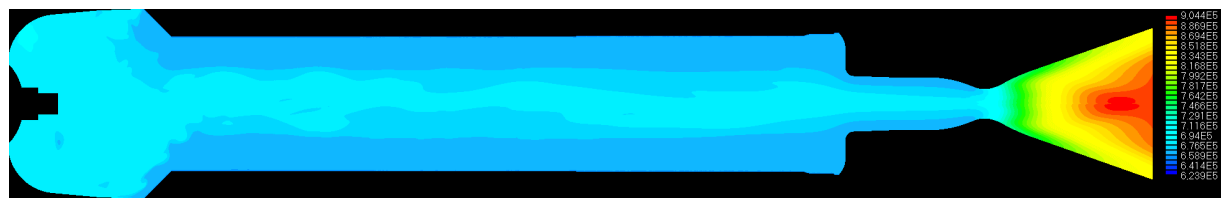

Рис. 8. Распределение энтропийной функции газовой фазы в плоскости $Y 0 Z$ : $a-N X=0, N Y=0, N Z=0 ; \sigma-N X=0, N Y=0,3, N Z=10 ; в-N X=0, N Y=20, N Z=10$

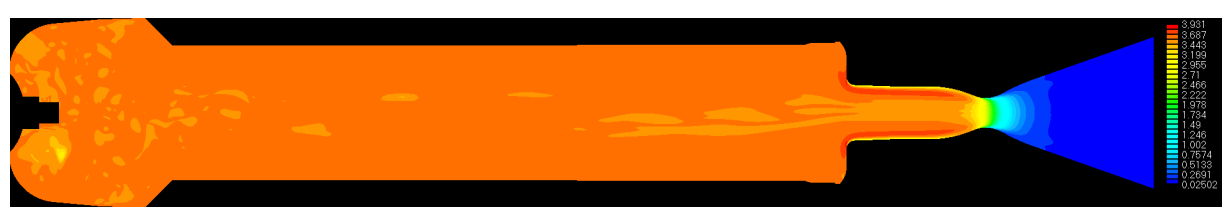

$a$

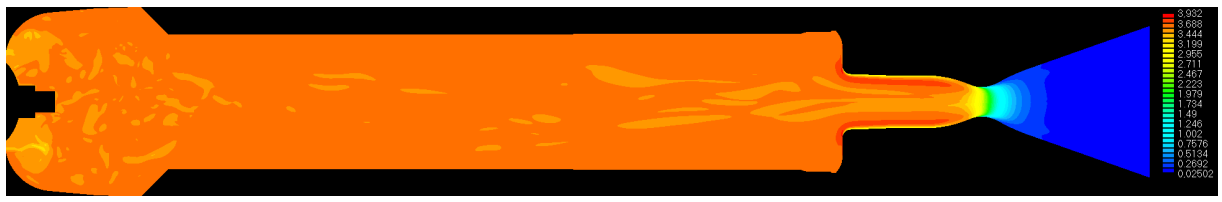

$\sigma$

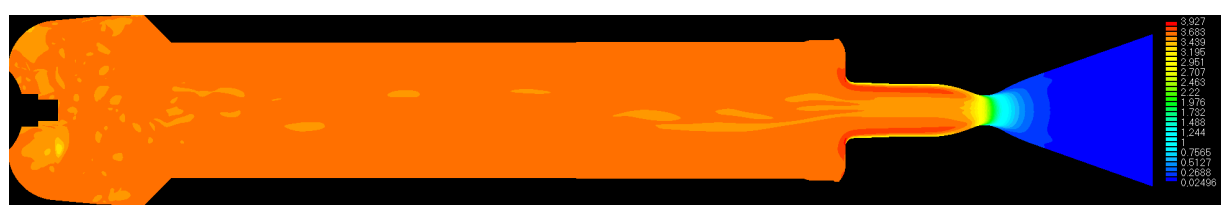

B

Рис. 9. Распределение плотности частиц малого диаметра в плоскости $Y 0 Z$ : $a-N X=0, N Y=0, N Z=0 ; \sigma-N X=0, N Y=0,3, N Z=10 ; \varepsilon-N X=0, N Y=20, N Z=10$

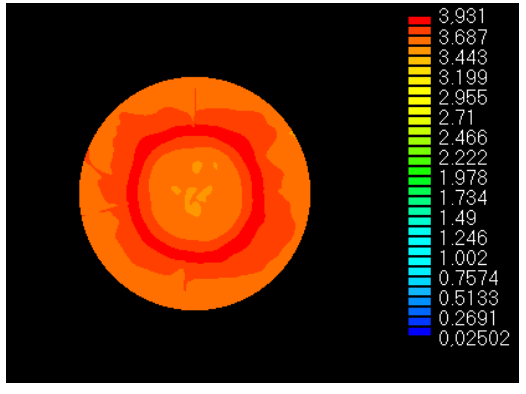

$a$

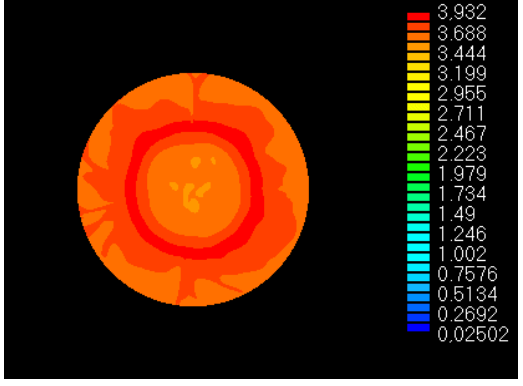

6

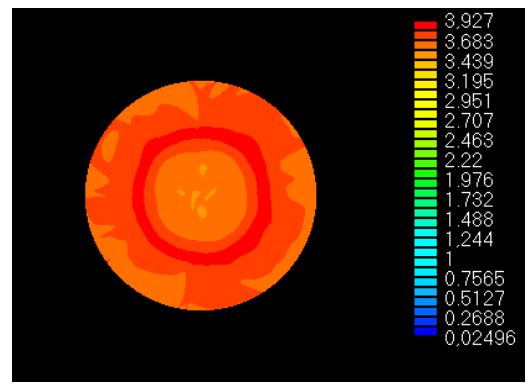

B

Рис. 10. Распределение плотности частиц малого диаметра в плоскости $X 0 Y$ : $a-N X=0, N Y=0, N Z=0 ; \sigma-N X=0, N Y=0,3, N Z=10 ; \varepsilon-N X=0, N Y=20, N Z=10$ 
больше уровень перегрузки, тем больше степень размазывания. Следует отметить также, что поток продуктов сгорания помнит исходную канально-щелевую форму поверхности горения заряда смесевого твердого топлива, изменяющуюся при выгорании. Как следствие, с учетом боковой составляющей перегрузки вдоль оси $0 Y(N Y)$ реализуется ассиметричное распределение плотности частиц по заднему днищу.

На рис. 11 показано распределение в камере сгорания и сопловом блоке РДТТ плотности мелкодисперсных несгоревших частиц (жидких капель) большого диаметра $\left(d_{4}=4\right.$ мкм $)$ в продуктах сгорания заряда смесевого твердого топлива (четвертая фаза гомогенно-гетерогенной смеси) в фиксированный момент времени в плоскости $Y 0 Z$ на тех же режимах ввода перегрузки, что и на рис. 7, 8 и 9. Дополнительно также на рис. 12 дано то же распределение, но в плоскости $X 0 Y$ в районе заднего днища. Характер распределения мелкодисперсных несгоревших частиц большего диаметра в целом остается тот же, что и характер распределения мелкодисперсных несгоревших частиц малого диаметра (см. рис. 9, 10). Однако степень изменения распределения плотности частиц (жидких капель) большего диаметра существенно выше, особенно в районе стенки заднего днища и стенки центрального предсоплового газохода.

Отметим, что результаты численного исследования хорошо согласуются с экспериментальными данными - результатами стендовых испытаний РДТТ и результатами летных испытаний крылатой ракеты, в состав которой входит ракетный двигатель. В результате летных испытаний крылатой ракеты на маршевом режиме работы РДТТ второй ступени, при траекторном маневрировании крылатой ракеты, зафиксирован локальный прогар заднего днища в районе его стыковки с центральным предсопловым газоходом. Результаты же стендовых испытаний этого РДТТ прошли штатно, без повреждений.

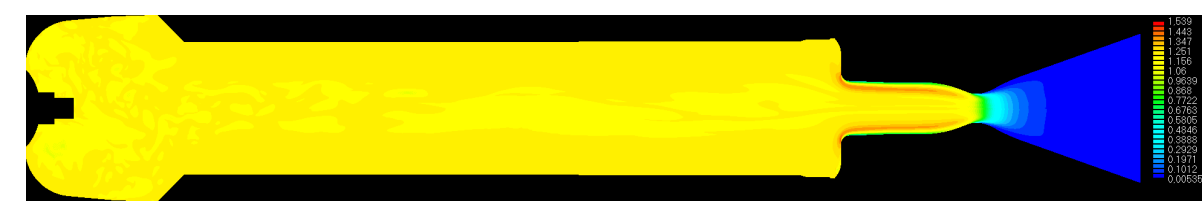

$a$

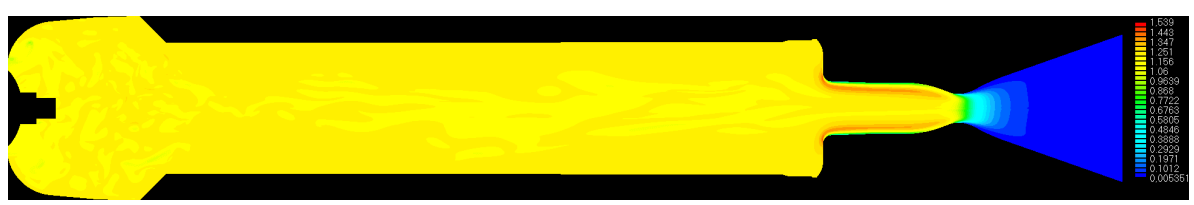

6

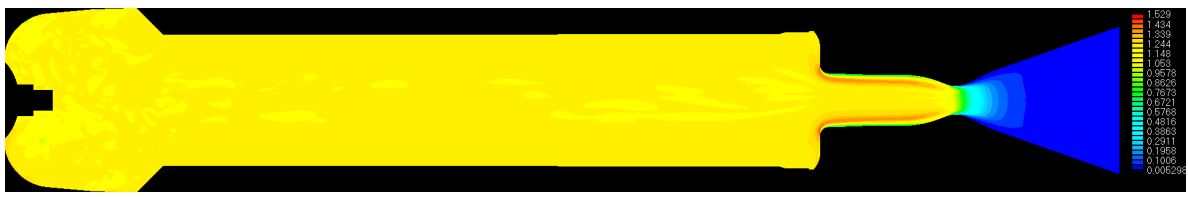

Рис. 11. Распределение плотности частиц большого диаметра в плоскости $Y 0 Z$ : $a-N X=0, N Y=0, N Z=0 ; \sigma-N X=0, N Y=0,3, N Z=10 ; \beta-N X=0, N Y=20, N Z=10$

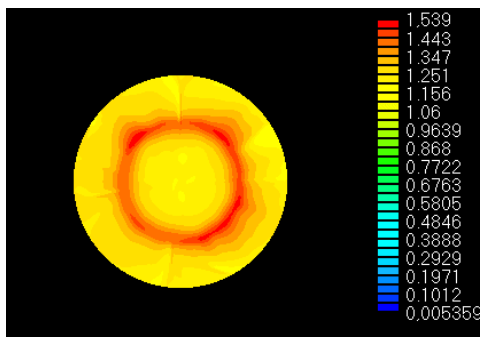

$a$

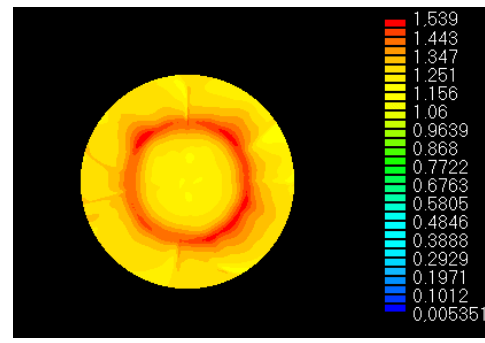

6

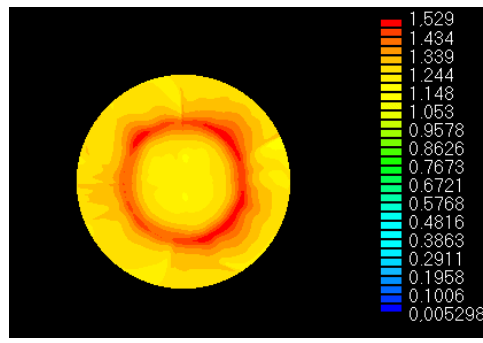

B

Рис. 12. Распределение плотности частиц большого диаметра в плоскости $X 0 Y$ : $a-N X=0, N Y=0, N Z=0 ; \sigma-N X=0, N Y=0,3, N Z=10 ; 6-N X=0, N Y=20, N Z=10$ 


\section{Заключение}

В заключение отметим, что разработанная методика расчета, приведенная в предыдущей статье, часть 1 , и полученная расчетная информация могут быть успешно использованы при проектировании и отработке новых образцов ракетной техники на твердом топливе с высокими тяговыми, массовыми, прочностными, шумовыми и другими эксплуатационными характеристиками.

Работа выполнена при финансовой поддержке Российского фонда фундаментальных исследований и администрачии Пермского края (грант № 19-41-590006-р-а) и отмечена дипломом 1-й степени ХХI Всероссийской НТК «Аэрокосмическая техника, высокие технологии и инновации - 2020».

\section{Библиографический список}

1. Давыдов Ю.М., Давыдова И.М., Егоров М.Ю. Влияние полетной перегрузки на неустойчивость рабочего процесса в камере сгорания ракетного двигателя на твердом топливе // Доклады академии наук. 2004. - T. 398, № 2. - C. 194-197.

2. Davydov Yu.M., Davydova In.M., Egorov M.Yu. Flight overloading effect on the working process instability in the combustion chamber of a solid-fuel rocket engine // Doklady Physics. Moscow. Nauka / Interperiodica. - 2004. - Vol. 49, no. 9. - P. 527-529.

3. Численное моделирование внутрикамерных процессов при выходе на режим работы ракетного двигателя твердого топлива / Г.Н. Амарантов, М.Ю. Егоров, С.М. Егоров, Д.М. Егоров, В.И. Некрасов // Вычислительная механика сплошных сред. - 2010. - Т. 3, № 3. - С. 5-17.

4. Давыдов Ю.М., Давыдова И.М., Егоров М.Ю. Неустойчивость рабочего процесса в двухкамерном ракетном двигателе на твердом топливе // Доклады академии наук. - 2011. - Т. 439, № 2. - С. $188-191$.

5. Численное моделирование нестационарных и нелинейных внутрикамерных процессов при срабатывании ракетного двигателя на твердом топливе специального назначения. Часть 1. Постановка вычислительного эксперимента / М.Ю. Егоров, С.М. Егоров, Д.М. Егоров, Р.В. Мормуль // Вестник Пермского национального исследовательского политехнического университета. Аэрокосмическая техника. 2016. - № 47. - С. 53-72.

6. Плотность атмосферы на различной высоте над землей [Электронный ресурс]. - URL: https://tehtab.ru/Guide/GuidePhysics/GuidePhysicsDensity/DensityAirHeight/ (дата обращения: 15.02.2021).

7. Иванов С.А. Газовая динамика: лаб. практикум [Электронный ресурс] / Самар. гос. техн. ун-т. Самара, 2014. - 62 c. - URL: http://physics.samgtu.ru/sites/physics.samgtu.ru/files/styles/ivanov_verstka.pdf (дата обращения: 15.02.2021).

8. Куренков В.И. Модели для оценки нагрузок, действующих на летательные аппараты [Электронный ресурc]. - URL: https://studfile.net/preview/7439596/ (дата обращения: 15.02.2021).

\section{References}

1. Davydov Yu.M., Davydova I.M., Egorov M.Yu. Vliyaniye polëtnoy peregruzki na neustoychivost rabochego protsessa $\mathrm{v}$ kamere sgoraniya raketnogo dvigatelya na tverdom toplive [The influence of flight overload on the instability of the working process in the combustion chamber of a solid-fuel rocket engine]. DOKLADY AKADEMII NAUK, 2004, vol. 398, No. 2, pp. 194-197.

2. Davydov Yu.M., Davydova In.M., Egorov M.Yu. Flight Overloading Effect on the Working Process Instability in the Combustion Chamber of a Solid-Fuel Rocket Engine // Doklady Physics. Moscow. Nauka / Interperiodica. 2004, no. 9, Vol. 49, pp. 527-529.

3. Amarantov G.N., Egorov M.Yu., Egorov S.M., Egorov D.M., Nekrasov V.I. Chislennoye modelirovaniye vnutrikamernykh protsessov pri vykhode na rezhim raboty raketnogo dvigatelya tvërdogo topliva [Numerical modeling of intra-chamber processes when entering the operating mode of a solid fuel rocket engine]. Computational continuum mechanics, 2010, Vol. 3, no. 3, pp. 5-17.

4. Davydov Yu.M., Egorov M.Yu. Numerical modeling of non-stationary transient processes in active and jet engines. Moscow: NAPN, 1999. 272 p.

5. Egorov M.Yu., Egorov S.M., Egorov D.M., Mormul R.V. Numerical modeling of non-stationary and nonlinear in-chamber processes during the operation of a special-purpose solid-fuel rocket engine. Part 1. Setting up a computational experiment. Bulletin of the Perm National Research Polytechnic University. Aerospace engineering. 2016. No. 47. pp. 53-72. 
6. Plotnost atmosfery na razlichnoy vysote nad zemley [Density of the atmosphere at different heights above the ground]. URL: https://tehtab.ru/Guide/GuidePhysics/GuidePhysicsDensity/DensityAirHeight/ (Date of access: 15.02.2021).

7. Ivanov S.A. Gazovaya dinamika. Laboratornyy praktikum [GAS DYNAMICS. Laboratory workshop]. Samara: Samara State Technical University, 2014, 62 p. URL: http://physics.samgtu.ru/sites/physics.samgtu.ru/files/styles/ivanov_verstka.pdf (Date of access: 15.02.2021).

8. Kurenkov V.I. Modeli dlya otsenki nagruzok, deystvuyushchikh na letatelnyye apparaty [Models for estimating loads acting on aircraft]. URL: https://studfile.net/preview/7439596/ (Date of access: 15.02.2021).

\title{
Об авторах
}

Егоров Михаил Юрьевич (Пермь, Россия) - доктор физико-математических наук, профессор, профессор кафедры «Высшая математика» ФГБОУ ВО ПНИПУ (614990, г. Пермь, Комсомольский пр., д. 29, e-mail: egorov-m-j@yandex.ru).

Егоров Дмитрий Михайлович (Пермь, Россия) - кандидат технических наук, первый заместитель генерального директора - главный конструктор АО НИИ полимерных материалов (614113, г. Пермь, ул. Чистопольская, д. 16, e-mail: egorovdimitriy@mail.ru).

Егоров Сергей Михайлович (Пермь, Россия) - кандидат физико-математических наук, начальник расчетного отдела АО НИИ полимерных материалов (614113, г. Пермь, ул. Чистопольская, д. 16, e-mail: know_nothing@bk.ru).

\begin{abstract}
About the authors
Mikhail Yu. Egorov (Perm, Russian Federation) - Doctor of Physical and Mathematical Sciences, Professor of Higher Mathematics Department FPMM, Perm National Research Polytechnic University (29, Komsomolsky av., Perm, 614990, Russian Federation; e-mail: egorov-m-j@yandex.ru).

Dmitry M. Egorov (Perm, Russian Federation) - Candidate of Technical Sciences, First Deputy General Director - Chief Designer, JSC "Scientific-Research Institute of Polymeric Materials" (16, Chistopolskaya st., Perm, 614113, Russian Federation; e-mail: egorovdimitriy@mail.ru).

Sergey M. Egorov (Perm, Russian Federation) - Candidate of Physical and Mathematical Sciences, Head of the Calculation Department, JSC "Scientific-Research Institute of Polymeric Materials" (16, Chistopolskaya st., Perm, 614113, Russian Federation; e-mail: know_nothing@bk.ru).
\end{abstract}

Получено 16.02.2021 